\title{
An Exploration of the Utilization of Electroencephalography and Neural Nets to Control Robots
}

\author{
Dan Szafir and Robert Signorile \\ Computer Science Department, \\ Boston College, Chestnut Hill, MA USA \\ \{szafird, signoril\}@bc.edu
}

\begin{abstract}
It has long been known that as neurons fire within the brain they produce measurable electrical activity. Electroencephalography (EEG) is the measurement and recording of these electrical signals using sensors arrayed across the scalp. The idea of Brain-Computer interfaces (BCIs), which allow the control of devices using brain signals, naturally present themselves to many extremely useful applications including prosthetic devices, restoring or aiding in communication and hearing, military applications, video gaming and virtual reality, and robotic control, and have the possibility of significantly improving the quality of life of many disabled individuals. The purpose of this research is to examine an off the shelf EEG system, the Emotiv EPOC@ System, as a costeffective gateway to non-invasive portable EEG measurements and to build a BCI to control a robot, the Parallax Scribbler®. We built middleware to interpret the outputs from the Emotiv and map them into commands for the Scribbler robot.
\end{abstract}

Keywords: Human-Robot Interaction, Computer Human Interface, Control Systems, Neural networks.

\section{Introduction}

Simple Brain-Computer interfaces (BCIs) currently exist and research and public interest in them only continues to grow. This research explores the process in creating a novel BCI that utilizes the Emotiv EPOC System to measure EEG waves to control the Parallax Scribbler robot. We wrote middleware to interpret the signals from the Emotive system and map those signals into commands for the Scribbler robot. These include commands such as move forward, move to an obstacle, etc. In latter sections of this paper, we describe the current research in BCI, describe the Emotiv system, the Scribbler robot, our middleware and a novel use of blinking (which can be detected by the Emotive systems) to enhance the command set for the robot [29].

\section{Electroencephalography}

EEG waves are created by the firing of neurons in the brain and were intensely in the fields of neuroscience and psychology [1] [2]. EEG waves are measured using 
electrodes attached to the scalp, which are sensitive to changes in postsynaptic potentials of neurons in the cerebral cortex. Postsynaptic potentials are created by the combination of inhibitory and excitatory potentials located in the dendrites. These potentials are created in areas of local depolarization or polarizations following the change in membrane conductance as neurotransmitters are released. These average of the potentials are amplified and combined to show rhythmic activity that is classified by frequency [3]. Electrodes are usually placed along the scalp as in Figure 1 [4]. One of the historical downsides of EEG measurement has been the corruption of EEG data by artifacts, which are electrical signals that are picked up by the sensors that do not originate from cortical neurons. One of the most common causes of artifacts is eye movement and blinking, however other causes exist [5]. Many EEG systems attempt to reduce artifacts and general noise by utilizing reference electrodes placed in locations where there is little cortical activity and attempting to filter out correlated patterns [6].

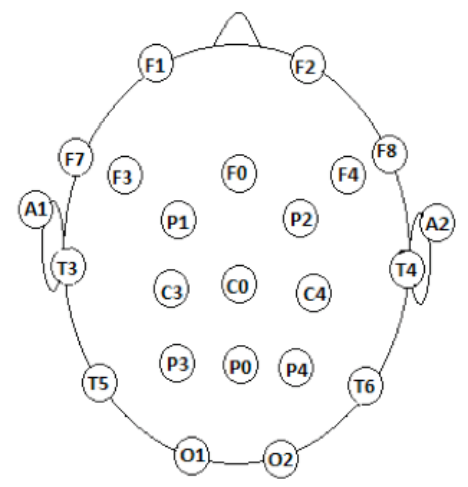

Fig. 1. Electrode Placement according to the International 10-20 System. Odd numbers on the right, even on the left. Letters correspond to lobes - F(rontal), T(emporal), P(arietal), and $\mathrm{O}$ (ccipital). C stands for Central (there is no central lobe).

\section{Brain-Computer Interfaces}

The term "Brain-Computer Interface" is the idea of linking the mind to computers [7]. The ultimate goal of BCI research is to create a system that is not only an "open loop" system that responds to users thoughts but a "closed loop" system that also gives feedback to the user. Researchers initially focused on the motor-cortex of the brain, the area which controls muscle movements, and testing on animals quickly showed that the natural learning behaviors of the brain could easily adapt to new stimuli as well as control the firing of specific areas of the brain [8] translate them into robotic activity [9][10] [11] [12]. Research is beginning to veer away from invasive BCIs due to the costly and dangerous nature of the surgeries required for such systems. Non-invasive alternatives for BCIs include EEG technology, Magnetoencephalography (MEG), and Magnetic Resonance Imaging (MRI), as well as the "partially invasive" Electrocorticography where sensors are placed within the skull but outside the gray matter of the brain. These methods are limited in that they are often susceptible to 
noise, have worse signal resolution due to distance from the brain, and have difficulty recording the inner workings of the brain. However, non-invasive techniques have the advantage of lower cost, greater portability, and the fact that they do not require any special surgery [13].

\section{Previous EEG BCI Research}

Though the idea of using EEG waves as input to BCIs has existed since the initial conception of BCIs, actual working BCIs based on EEG input have only recently appeared [14]. Most EEG-BCI systems follow a similar paradigm of reading in and analyzing EEG data, translating that data into device output, and giving some sort of feedback to the user (Figure 2), however implementing this model can be extremely challenging [15]. The primary difficulty in creating an EEG-based BCI is the feature extraction and classification of EEG data that must be done in real-time if it is to have any use.

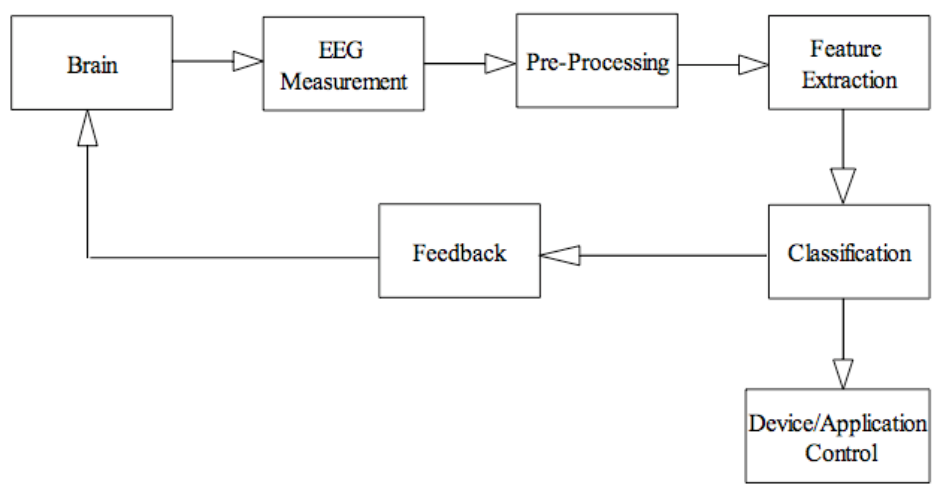

Fig. 2. Brain-Computer Interface Design Pattern

Feature extraction deals with separating useful EEG data from noise and simplifying that data so that classification, the problem of trying to decide what the extracted data represents, can occur. There is no best way of extracting features from EEG data and modern BCIs often use several types of feature extraction including Hjorth, wavelet transforms and Fourier transforms. The major features that EEG-BCI systems rely on are event-related potentials (ERPs) and event-related changes in specific frequency bands [16][17]. BCI systems are further complicated by the fact that there is no standard way of classifying the extracted data. Various types of pattern recognizers are employed to try to match the input data to known categories of EEG archetypes [18]. Researchers have also relied on unsupervised learning algorithms to find natural clusters of EEG segments that are indicative of certain kinds of mental activities with varying degrees of success [19][20]. Feedback is essential in BCI systems as it allows users to understand what brainwaves they just produced and to learn behavior that can be effectively classified and controlled [21]. 
EEG-BCIs can be classified as either synchronous or asynchronous. The computer drives synchronous systems by giving the user a cue to perform a certain mental action and then recording the user's EEG patterns in a fixed time-window. Asynchronous systems are driven by the user and operate by passively and continuously monitoring the user's EEG data and attempting to classify it on the fly. Synchronous protocols are far easier to construct and have historically been the primary way of operating BCI systems [22] [23][24].

\section{Our Research Project: Combining Machine learning, Neural Nets, Brain Waves and Our Middleware to Control Personal Robots}

The goal of our project was to investigate and suggest the use of brainwaves to control personal robots (and thus demonstrate the more general proposition that robots can be controlled by brainwaves). Using training of the Emotiv System, we were able to extract the EEG signals from the headset, categorize them into one of several groups, translate that group to a robotic command, and finally control the robot. The two major hardware components of our system are the Emotiv Headset and the Scribbler robot. The next subsections briefly describe each system. Then we discuss our integration of the two systems sand finally we describe some adjustments we made to extend the overall system.

\subsection{The Emotiv@ System}

The Emotiv System is based around the EPOC headset for recording EEG measurements and software suit which processes and analyzes the data. This research originally uses the Research Edition of this off the shelf product. The Research Edition includes the Emotiv Control Panel, EmoComposer (an emulator for simulating EEG signals), EmoKey (a tool for mapping various events detected by the headset into keystrokes), TestBench, which enables the capture of raw EEG data from each individual sensor [26].The Emotiv system can measure engagement/boredom, frustration, meditation, instantaneous excitement, and long-term. The Cognitiv suite can measure 13 active thoughts as well as the passive neutral state. This software works by running the input from the electrodes through a neural network and attempting to classify the signals as one of the 13 built-in "prototype" action thoughts.The core of the Emotiv SDK is the "EmoEngine," which is a logical abstraction that "communicates with the Emotiv headset, receives preprocessed EEG and gyroscope data, manages user-specific or application-specific settings, performs post-processing, and translates the Emotiv detection results into an easy-to-use structure called an EmoState." Every EmoState represents the current input from the headset including "facial, emotional, and cognitive state" contains electrode measurements for each contact. Utilizing the Emotiv API consists of connecting to the EmoEngine, detecting and decoding new EmoStates, and calling code relevant to the new EmoState (Figure 3). 


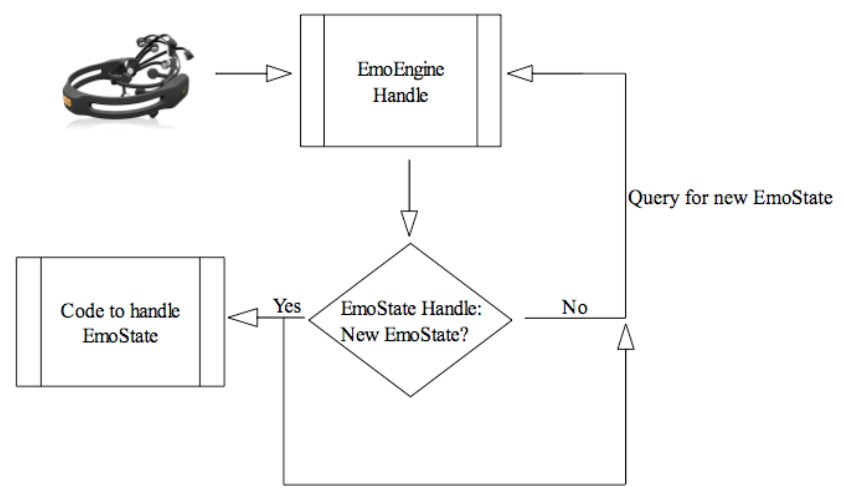

Fig. 3. High-level View of the Utilization of Emotiv

\subsection{The Parallax Scibbler ${ }^{\circledR}$ Robot and IPRE Fluke}

The Parallax Scribbler robot is a fully assembled reprogrammable robot built around the BASIC Stamp® 2 microcontroller. It contains built in photovoltaic sensors, infrared sensors, line sensors, two independent DC motors to drive the two wheels, three LED lights, a speaker, and a serial port[28]. The Institute for Personal Robots in Education (IPRE) Fluke is an add-on board created by Georgia Robotics that plugs into the Scribbler's serial port and adds color vision, IR range sensing, internal voltage sensing, an extra LED, and bluetooth functionality and has created the Myro APIs to control the Scribbler using Python [29].

\subsection{Control Implementation}

The code implementing this control scheme is divided into four basic parts: connecting to the Emotiv headset via the Emotiv API, connecting to the Scribbler through the

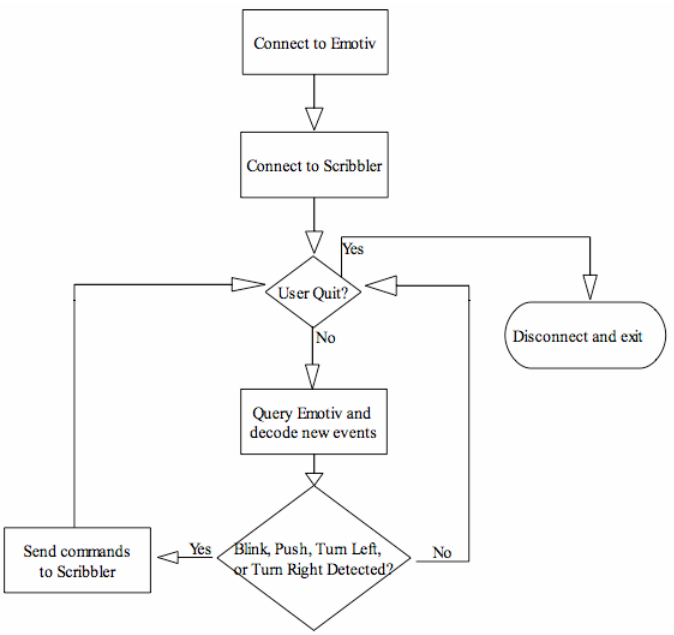

Fig. 4. High-level View of the Control Scheme 
Myro Python libraries, reading and decoding Emotiv events and sending the corresponding commands to the Scribbler, and closing the connections when the user is done (Figure 4).

\subsection{Decoding and Handling EmoStates}

There are four major steps in reading and decoding information from the EPOC headset: creating the EmoEngine and EmoState handles, querying for the most recent EmoState, deciding if this is a new EmoState, and decoding the EmoState. The EmoEngine handle allows for queries to get direct input from the headset including contact quality, raw electrode input, and the connection quality. New EmoStates are constantly created by the EmoEngine which represent recognized actions such as facial expressions, changed emotional status, and detected thoughts and can be queried through the EmoState handle.Next the program determines the event type returned by the EmoEngine. There are three categories of event types: hardware-related events, new EmoState events, and suite-related events. Once we have decoded which thought sparked the EmoState, we send the appropriate call to the Scribbler (Push $\rightarrow$ Move Forward, Turn Left $\rightarrow$ Turn Left, Turn Right $\rightarrow$ Turn Right). We initially experimented with using the power of the thought as an input to the power of the robotic action, however we found that this control scheme was too difficult to use and it ended up being far more intuitive to use specific values for turning and moving forward no matter the thought-power. This allowed the user to concentrate on the thoughts alone and not have to additionally worry about how "hard" to think about the thoughts.

The internal sampling rate in the EPOC headset is $2048 \mathrm{~Hz}$ which is filtered to remove artifacts and alias frequencies own to about $128 \mathrm{~Hz}$. Any given motion input to the Scribbler using Bluetooth takes approximately 2 seconds to execute, while picture taking take slightly longer as it has to capture and send data back. Thus we needed to synchronize the sampling rate with the actual Scribbler command execution (so that commands are not just queued up, but executes in a orderly fashion). To solve this problem, we introduced a sampling variable to only decode one in every ten input EmoStates to limit the EmoStates created. Using this sampling variable we filter out those extra states that really only correspond to one event by using a sample rate small enough that it will still capture events which send more than 10 input EmoStates while sending only one command to the Scribbler instead of queuing up 10. This system worked much better, and even had the added bonus of filtering out "noise" thoughts when the headset detected a push or turn thought for a fraction of a second.

To extend the number of command we can send to the Scribbler, we created an additional mode, which remaps the same input thoughts to different outputs in the robot. This is hugely beneficial as it does not increase the difficulty in recognizing new thoughts and also does not require the user to train additional thoughts, thus giving double the usability with only one additional input. This additional input is raising the eyebrows, which toggle between the original and the new mode. We decided on utilizing the raising of eyebrows as a toggle as it is very easily trained and accurately recognized by the headset. The addition of more modes is certainly possible and is an excellent way of adding functionality without adding the cost of recognizing and learning new thoughts. In the end, it was completely feasible to control the Scribbler robot using the EPOC headset proving the viability of EEG based BCI technology. 


\subsection{Blink Detection and Data Reduction/Noise Reduction}

We next decided to explore the Pre-Processing, Feature Extraction, and Classification of EEG data by analyzing eye blinks. The analysis of eye blinks is useful in BCI development for two reasons: eye blinks can be used as control inputs and if they are not they must be filtered out lest they corrupt the useful EEG data. We decided on eye blinks since they are immediately recognizable in one particular channel from the headset. This allowed us to immediately reduce the amount of input data by a factor of 14 since we could discount the other 13 input channels.

Reducing the size of the data set is the primary focus of pre-processing and feature extraction whose goal is to get rid of extraneous and noisy data while preserving data that can best differentiate between classes. We recorded twenty, ten second clips, ten of which we blinked during and ten of which we didn't. We then exported the clips to MATLAB. These recordings produced a lot of data because in addition to the 14 EEG channels capturing electrode data at $128 \mathrm{~Hz}$ the headset also records gyroscope data, battery data, packet information, etc. and each 10 second clip ended up had roughly 36000 data points and combined I recorded 720000 data points.

The first step of our feature extraction was to use just the channel where blinks are clearly visible. The classification using MATLABS' nprtool to create a two-layer feedforward neural network with backpropagation obtained only a $65 \%$ accuracy. Though there was a certain pattern to the blinks, the neural net was thrown off because the blinks were not normalized with respect to time. The neural net treated time as an attribute, and thus did not classify two samples that both contain blinks but where the blinks occur at different times. Time was correlated to blinks in respect to how long the blink takes and thus how wide the blink spike will be, however the time that the blink occurs is not a usable attribute.

To solve this problem, we decided to further reduce the amount of data the neural net worked with along with normalizing any blinks found. Recognizing that blinks correlate to spikes in EEG data, we scanned each 10-second clip looking for the largest spikes. We found that blinks typically were represented by a surge in the 4500 to 4800 $\mu$ volt range over approximately .59 seconds and were followed by a characteristic dip of around $50 \mu$ volts over approximately .20 seconds. This pattern was very clear and easily distinguishable from a non-blink state; we first noticed it when applying unsupervised K-Means Clustering to detect naturally occurring patterns in the data.We used this information to further filter each of the 20 inputs down to 1.6-second segments, each of which highlighted the maximum spike of the original ten-second segment. This normalized the blinks by having each blink start at roughly the same time and additionally filtered out noise that was unrelated to the blinks creating data that was much easier for a neural net to distinguish. Using these inputs, neural net accuracy improved to $100 \%$, however we wanted to see if this system truly recognized blinks or was over-trained on the input data. We then recorded five more segments, 3 with blinks and 2 without, and followed the same pre-processing/feature extraction steps and fed the data to the neural net. The neural net accurately predicted all of these new inputs even though it had not been trained upon them, showcasing that it was truly was extendable and actually recognizing blink patterns. These are very promising results that prove the feasibility of utilizing a neural net to classify blinks, however it 
would be best to obtain a larger sample size to accurately test the classification performance of this scheme. Now blinks could be used to effectively double our thoughts (think of the recognizable blink as a "shift" key on a keyboard).

\section{Conclusions}

Part of our research was to examine the state of EEG-based BCI construction and implementation. In particular, we wanted to test the feasibility of BCI to control personal items such as a personal robot. Our investigation demonstrates that, with our middleware, it is a feasible system that will likely only improve and become more widespread in the future. The system we constructed was largely a success as we were able to create a system whereby we could control a robot with our thoughts and we further created accurate blink-recognizing software to enhance the amount of thoughts we can recognize.Furthermore, our system showcases the possibilities of BCI's in aiding the disabled. For instance, a person who could only move their head could certainly use our system to control a motorized wheelchair accurately using their thoughts. In addition, had they a computer built into the headset, they could easily switch modes by raising their eyebrows and then use their thoughts as an input to the computer, by using the same thoughts that had moved the wheelchair to control the mouse and double-blinking to click. An alternative would be to keep the thoughts controlling the wheelchair while utilizing the gyroscope in the headset to control the mouse, enabling the user to have simultaneous control of the wheelchair and computer. Further research can certainly lead to direct implementation of such systems and can explore the recognition of thoughts beyond those included in the Emotiv API.

\section{References}

1. Swartz, B.E., Goldensohn, E.: Timeline of the history of EEG and associated fields. Electroencephalography and Clinical Neurophysiology 106, 173-176 (1998)

2. Millett, D.: Hans Berger: from psychic energy to the EEG. Perspectives in Biology and Medicine 44(4), 522-542 (2001)

3. Nunez, P.L., Srinivasan, R.: Electric Fields of the Brain: The Neurophysics of EEG. Oxford University Press, Oxford (1981)

4. Niedermeyer, E., da Silva, F.L.: Electroencephalography: Basic Principles, Clinical Applications and Related Fields, 5th edn., p. 140. Lippincott Williams \& Wilkins (2005)

5. Rowan, A.J.: Primer of EEG. Elsevier Science, Philadelphia (2003)

6. Ludwig, K.A., et al.: Employing a Common Average Reference to Improve Cortical Neuron Recordings from Microelectrode Arrays. Journal of Neurophysiology, September 3 (2008)

7. Vidal, J.: Toward Direct Brain-Computer Communication. Annual Review of Biophysics and Bioengineering 2, 157-180 (1973)

8. Fetz, E.E.: Operant Conditioning of Cortical Unit Activity. Science 163, 955-958 (1969)

9. Kennedy, P.R., et al.: Activity of single action potentials in monkey motor cortex during long-term task learning. Brain Research 760(1-2), 251-254 (1997)

10. Wessber, J., et al.: Real-time prediction of hand trajectory by ensembles of cortical neurons in primates. Nature 408(6810), 361-365 (2000) 
11. Carey, B.: Monkeys Think, Moving Artifiacl Arm as Own. The New York Times, May 29 (2008)

12. Hochberg, L.R., et al.: Neuronal ensemble control of prosthetic devices by a human with tetraplegia. Nature 442, 164-171 (2006)

13. Fabiani, G.E., et al.: Conversion of EEG activity into cursor movement by a brain-computer interface

14. Vidal, J.: Toward Direct Brain-Computer Communication

15. Omidvarnia, A.H., et al.: Kalman Filter Parameters as a New EEG Feature Vector for BCI Applications

16. Niedermeyer: Electroencephalography, pp. 1265-1266

17. Sellers, E.W., et al.: A P300 event-related potential brain-computer interface (BCI): The effects of matrix size and inter stimulus interval on performance. Biological Psychology 73(3), 242-252 (2006)

18. Adlakha, A.: Single Trial EEG Classification. Swiss Federal Institute of Technology, July $12(2002)$

19. Lu, S., et al.: Unsupervised Brain Computer Interface based on Inter-Subject Information. In: 30th Annual International IEEE EMBS Conference, Vancouver, British Columbia, Canada, August 20-24 (2008)

20. Niedermyer: Electroencephalography, p. 1240

21. Kauhanen, L., Palomaki, T., Jylanki, P., Aloise, F., Nuttin, M., Millan, J.R.: Haptic feedback compared with visual feedback for BCI. In: Kauhanen, L., Palomaki, T., Jylanki, P., Aloise, F., Nuttin, M., Millan, J. (eds.) Proceeding of 3rd International BCI Workshop and Training Course 2006, Graz, Austria, September 21-25, pp. 66-67 (2006)

22. Niedermeyer: Electroencephalography, p. 1265

23. Birbaumer, N., et al.: The Thought Translation Device (TTD) for Completely Paralyzed Patients. IEEE Transactions on Rehabilitation Engineering 8(2), 190-193 (2000)

24. Galán, F., et al.: A Brain-Actuated Wheelchair: Asynchronous and Non-invasive BrainComputer Interfaces for Continuous Control of Robots. Clinical Neurophysiology 119(9), 2159-2169 (2008)

25. Drummond, K.: Pentagon Preps Soldier Telepathy Push. Wired Magazine, May 14 (2009)

26. Emotiv Website, http: / /www. emotiv.com

27. The Scribbler: A Reprogrammable Robot, http://www.parallax.com/tabid/455/Default.aspx, Copyright 2010 by Parallax Inc. (accessed November 4, 2010)

28. Usage Guides, http://www.roboteducation.org/guides.html, Copyright (C) 2007 Institute for Personal Robots in Education (accessed November 4, 2010)

29. Szafir, D.: Non-Invasive BCI through EEG, unpublished Undergraduate Honors Thesis in Computer Science, Boston College (2010) 